\section{Insulin-like signaling and the neural circuit for integrative behavior in C. elegans}

\author{
Eiji Kodama, ${ }^{1}$ Atsushi Kuhara, ${ }^{1}$ \\ Akiko Mohri-Shiomi, ${ }^{1,4}$ Koutarou D. Kimura, ${ }^{1,5}$ \\ Masatoshi Okumura, ${ }^{1}$ Masahiro Tomioka, ${ }^{2}$ \\ Yuichi Iino, ${ }^{2}$ and Ikue Mori ${ }^{1,3,6}$ \\ ${ }^{1}$ Division of Biological Science, Graduate School of Science, \\ Nagoya University, Nagoya 464-8602, Japan; ${ }^{2}$ Molecular \\ Genetics Research Laboratory, The University of Tokyo, \\ Tokyo 113-0033, Japan; ${ }^{3}$ Institute for Advanced Research, \\ Nagoya University, Nagoya 464-8602, Japan
}

Caenorhabditis elegans exhibits a food-associated behavior that is modulated by the past cultivation temperature. Mutations in INS-1, the homolog of human insulin, caused the defect in this integrative behavior. Mutations in DAF-2/insulin receptor and AGE-1/phosphatidylinositol 3 (PI-3)-kinase partially suppressed the defect of ins-1 mutants, and a mutation in DAF-16, a forkhead-type transcriptional factor, caused a weak defect. In addition, mutations in the secretory protein HEN-1 showed synergistic effects with INS-1. Expression of AGE-1 in any of the three interneurons, AIY, AIZ, or RIA, rescued the defect characteristic of age-1 mutants. Calcium imaging revealed that starvation induced INS-1-mediated down-regulation of AIZ activity. Our results suggest that INS-1, in cooperation with HEN-1, antagonizes the DAF-2 insulin-like signaling pathway to modulate interneuron activity required for food-associated integrative behavior.

Supplemental material is available at http://www.genesdev.org.

Received August 9, 2006; revised version accepted September $18,2006$.

The secreted peptide hormone insulin modulates neural plasticity. Insulin and insulin receptors are expressed in several regions of the rat brain (Havrankova et al. $1978 \mathrm{a}, \mathrm{b}$ ), insulin receptors localize to post-synapses (Abbott et al. 1999), and insulin can produce long-term depression (LTD) of synaptic transmission through endocytosis of $\alpha$-amino-3-hydroxy-5-methyl-4-isoxazolepropionic acid (AMPA) receptors in rat hippocampal CA1 neurons (Man et al. 2000). In addition, Phosphatidylinositol 3 (PI-3)-kinase that functions in the insulin signaling pathway is thought to induce long-term potentiation (LTP) of synaptic transmission in the dentate gyrus of rat

[Keywords: C. elegans; thermotaxis; learning and memory; integrative behavior; insulin; neural circuit]

Present addresses: ${ }^{4}$ University of Texas Health Science Center at Houston, Department of Microbiology and Molecular Genetics, Houston, TX 77030, USA; ${ }^{5}$ Structural Biology Center, National Institute of Genetics, Mishima 411-8540, Japan.

${ }^{6}$ Corresponding author.

E-MAIL m46920a@nucc.cc.nagoya-u.ac.jp; FAX 81-52-789-4558.

Article is online at http://www.genesdev.org/cgi/doi/10.1101/gad.1479906.
(Kelly and Lynch 2000). These alterations by proteins of the insulin signaling pathway may be involved in learning and memory, but what kind of behavior the insulin signaling pathway modulates has been largely unknown.

The nematode Caenorhabditis elegans is well suited for the analysis of the molecular and cellular mechanisms underlying neural plasticity because of its accessible genetics, stereotyped behavioral responses, and its simple nervous system consisting of 302 neurons whose connections are entirely known (White et al. 1986). Recently, physiological analysis of the neural circuit in live worms has become possible by the use of cameleon, a genetically encodable calcium indicator, to measure $\mathrm{Ca}^{2+}$ concentration changes (Miyawaki et al. 1997; Kimura et al. 2004).

C. elegans exhibits thermotaxis, an integrative behavior in which well-fed animals in a thermal gradient are attracted to their cultivation temperature, whereas starved animals avoid it (Hedgecock and Russell 1975; Mohri et al. 2005; Rankin 2005). This food-associated behavioral plasticity, regarded the most complex behavior in C. elegans, is an ideal behavioral paradigm for comprehensive study of neural plasticity at the molecular, physiological, and behavioral levels. In this study, we show that in cooperation with a secreted protein HEN-1, an insulin homolog INS-1, and insulin-like signaling pathway modulate neuronal activity of interneurons to execute thermotaxis behavior in C. elegans. We suggest that a neuroendocrine system is important for modulating the neural circuit that underlies neural plasticity.

\section{Results and Discussion}

Insulin homolog INS-1 is required for food-associated temperature responsive behavior

Wild-type animals cultivated at $17^{\circ} \mathrm{C}$ in well-fed conditions migrated to the center of the agar plate, which corresponded to their cultivation temperature (Fig. 1A,C,K). By contrast, most of the wild-type animals cultivated at $17^{\circ} \mathrm{C}$ for $3 \mathrm{~h}$ in food-deprived conditions avoided their cultivation temperature (Fig. 1D,K). Similarly, wild-type animals cultivated at $25^{\circ} \mathrm{C}$ with food migrated to their cultivation temperature (Fig. 1G,L), whereas most avoided their cultivation temperature after being cultivated at $25^{\circ} \mathrm{C}$ without food for $1 \mathrm{~h}$ (Fig. $\left.1 \mathrm{H}, \mathrm{L}\right)$. These results indicate that $C$. elegans can associate cultivation temperature with feeding state and memorize this information (Hedgecock and Russell 1975; Mohri et al. 2005).

Previous studies have identified the neural circuit and several genes required for thermotaxis (Mori and Ohshima 1995; Komatsu et al. 1996; Ishihara et al. 2002; Kuhara et al. 2002; Murakami et al. 2005; Okochi et al. 2005; Inada et al. 2006). However, how C. elegans executes thermotaxis remains to be understood at the molecular and physiological levels. The aho-2(nj32) mutant was isolated in our genetic screen for mutants defective in this integrative behavior (Mohri et al. 2005). aho2(nj32) mutants always migrated to their cultivation temperature after starvation as well as after feeding (Fig. $1 \mathrm{E}, \mathrm{F}, \mathrm{I}-\mathrm{L})$; we designated this phenotype as an Abnormal hunger orientation (Aho) phenotype. The Aho phenotype of $a$ ho-2(nj32) mutants could result from a defect either 
A

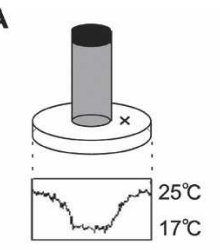

B

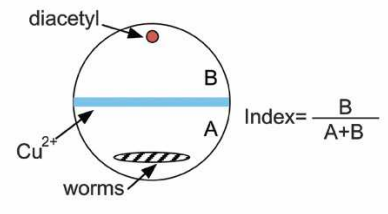

$17^{\circ} \mathrm{C}$ - cultivated

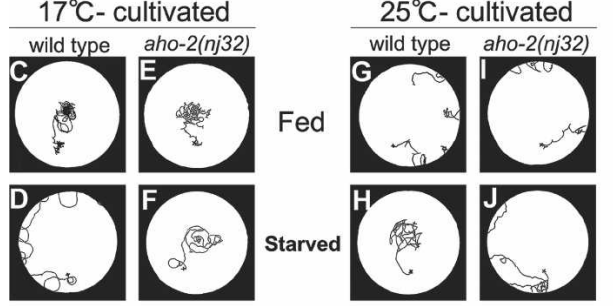

K $\quad 17^{\circ} \mathrm{C}$ - cultivated
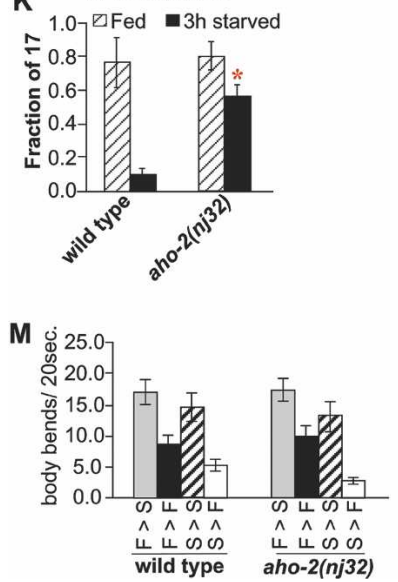

$\mathbf{N}$

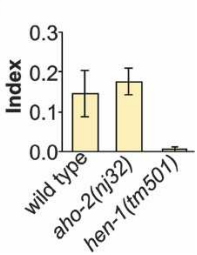

Figure 1. aho-2(nj32) shows defects in food-associated thermotactic behavioral plasticity, normal response to starvation, and normal integration of two different chemosensory inputs. $(A$, top $)$ Thermotaxis assay system. Single animals were placed on the agar at $\sim 22^{\circ} \mathrm{C}$, as indicated by the cross. (Bottom) A thermograph shows the stable radial temperature gradient $\left(17^{\circ} \mathrm{C}-25^{\circ} \mathrm{C}\right)$ of the dish surface. $(B) \mathrm{In}-$ teraction assay system. $(C-J)$ Tracks on a radial temperature gradient of individual animals cultivated at $17^{\circ} \mathrm{C}$ or $25^{\circ} \mathrm{C}$ with or without food. $(K)$ Results of the thermotaxis assay for well-fed or starved animals cultivated at $17^{\circ} \mathrm{C}$. Thermotaxis was evaluated using "Fraction of 17," which includes the class "17." The asterisks indicate $p<0.005$, as identified by $t$-tests in a comparison of starved aho2(nj32) mutants with starved wild-type animals. Fed animals, $n \geq 30$; starved animals, $n \geq 60$. Error bars indicate SEM. $(L)$ Results of the thermotaxis assay for animals cultivated at $25^{\circ} \mathrm{C}$. Thermotaxis was evaluated using "Fraction of 25," which includes the class "25." The asterisks indicate $p<0.005$ in a comparison of aho2(nj32) mutants with starved wild-type animals ( $n \geq 68$ animals). Error bars indicate SEM. $(M)$ Modulation of the locomotory rate. The number of body bends in $20 \mathrm{sec}$ on the assay plates was scored. Gray and black bars indicate the results for well-fed animals transferred to assay plates without food $(\mathrm{F}>\mathrm{S})$ and with food $(\mathrm{F}>\mathrm{F})$, respectively. Hatched and white bars indicate the results for starved animals transferred to assay plates without food $(\mathrm{S}>\mathrm{S})$ and with food $(\mathrm{S}>\mathrm{F})$, respectively $(n=45$ animals). Error bars indicate SD. $(N)$ Wild-type and aho-2(nj32) animals were tested in the interaction assay, and the results are represented as Index $(\mathrm{B} /[\mathrm{A}+\mathrm{B}])$.

in association between cultivation temperature and starvation or in recognition of starvation per se. To address these possibilities, we tested the responses of aho2(nj32) animals to changes in feeding state using a locomotory activity assay (Sawin et al. 2000). Well-fed wildtype animals move more slowly in plates with food than

without food, and starved wild-type animals move even more slowly in plates with food than do well-fed animals (Fig. 1M). aho-2(nj32) and wild-type animals exhibited nearly the same responses to changes in feeding state (Fig. 1M), suggesting that aho-2(nj32) animals can respond to starvation and exhibit a defect in association between cultivation temperature and starvation (Mohri et al. 2005).

We investigated whether aho-2(nj32) mutants had a defect in integration of different chemosensory inputs using an interaction assay (Fig. 1B) that is a behavioral test for the integration of two opposing signals, a signal from an attractive odorant, diacetyl, and from a repulsive metal, $\mathrm{Cu}^{2+}$ ion (Ishihara et al. 2002). HEN-1 is a secretory protein with an LDL receptor motif and is required for the behavioral task tested in this interaction assay (Ishihara et al. 2002). In contrast to hen-1(tm501)-null mutants, aho-2(nj32) mutants responded normally (Fig. 1N). aho-2(nj32) mutants exhibited normal responses to diacetyl and $\mathrm{Cu}^{2+}$ ions separately (data not shown), implying that the integrative process of the two chemical compounds is normal in aho-2(nj32) mutants.

The gene aho-2 was mapped to the 0.08 map unit region in the center of chromosome IV (Mohri et al. 2005; data not shown), which is covered by the three cosmids. We found that only F13B12 rescued the defect of aho2(nj32) (Fig. 2A). Among six predicted genes in the region covered by F13B12 (data not shown), a PCR product containing ins-1, the C. elegans gene most closely related to human insulin among 38 insulin-related genes (Pierce et al. 2001; Li et al. 2003), rescued the defect of the aho2(nj32) mutant (Fig. 2A). The ins-1(nr2091) mutant, a previously isolated putative null mutant (Pierce et al. 2001), also showed an Aho phenotype (Fig. 2C). We found a 130-base-pair (bp) deletion from the first exon to the second exon in ins-1 of aho-2(nj32) mutants (data not shown). These results led us to conclude that aho-2 is identical to ins-1.

\section{Neuronal expression of INS-1 is important for integrative thermotaxis behavior}

We observed ins-1-expressing cells using an ins-1 promoter::GFP fusion gene. As previously reported, fluorescence was observed in many head neurons, including ADF, AIA, AIM, ASE, ASG, ASH, ASI, ASJ, AWA, BAG, and NSM, and was also observed in the intestine, hypodermis, and vulval muscle (Fig. 2E; Pierce et al. 2001; data not shown).

Expressing ins-1 cDNA from its own promoter or in all neurons using the unc-14 promoter almost fully rescued the Aho phenotype of ins-1(nr2091) mutants, whereas no rescue occurred when expressing the ins-1 cDNA in intestine using the ges-1 promoter (Fig. 2C). These results indicate that neuronal expression of INS-1 is sufficient to rescue the food-associated thermotactic behavior defect of ins-1(nr2091) mutants. We conducted cell-specific rescue experiments to determine if the expression of INS-1 in any particular neuron is required for association between temperature and feeding state. The expression of ins-1 cDNA using the ins-1, ncs-1, lin-11, unc-86, and ceh-14 promoters effectively rescued the defect of ins-1(nr2091) mutants, and weak rescue occurred upon ins- 1 cDNA expression from osm- 6 and gpa-2 promoters (Fig. 2D). In contrast, no rescue occurred upon expression of ins-1 cDNA using the unc-42, tph-1, gcy-8, glr-3, or 


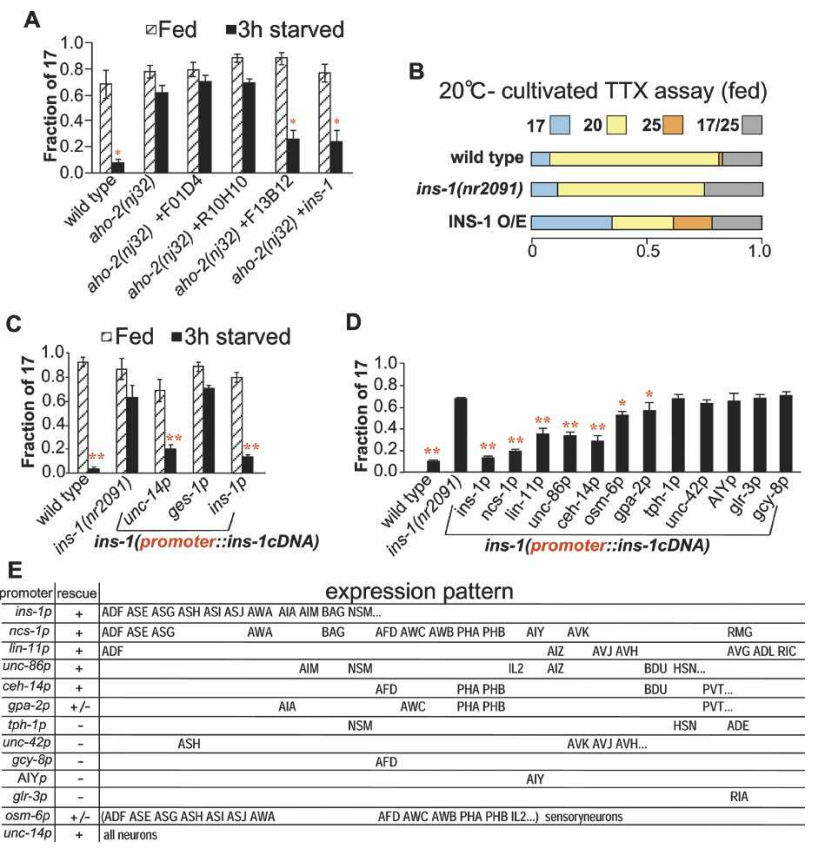

Figure 2. INS-1 is required in neurons for integrative thermotactic behavior. (A) Rescue experiments for the food-associated thermotactic behavior defect in aho-2(nj32) mutants. The asterisks indicate $p<0.05$ for a comparison with starved aho-2(nj32) mutants. Fed animals, $n \geq 30$; starved animals, $n \geq 60$. Error bars indicate SEM. (B) The thermotaxis behavior of animals cultivated at $20^{\circ} \mathrm{C}$ with food. The phenotypic categories are described in the Supplemental Material. The data, presented as horizontal stacked bar graphs, represent the average of three independent thermotaxis assays using 20 animals per assay. Three independent transgenic lines were tested for the INS-1 overexpression (O/E) line (containing $100 \mathrm{ng} / \mathrm{\mu L}$ ins-1 genomic DNA); $n=60$ for wild type, ins-1(nr2091), and each INS-1 $\mathrm{O} / \mathrm{E}$ line. $(C, D)$ Rescue experiments of the food-associated thermotactic behavior defect in the ins-1(nr2091) mutant. Single and double asterisks indicate $p<0.05$ and $p<0.01$, respectively, for a comparison with starved ins-1(nr2091) mutants. Fed animals, $n \geq 30$; starved animals, $n \geq 60$. Error bars indicate SEM. (E) Summary of results of the rescue experiments for ins-1(nr2091) and neuronal expression pattern of INS-1 with different promoters.

AIY promoter (Fig. 2D). Essentially, we did not identify any single neuron where the expression of INS-1 was required for the rescue, suggesting that INS-1 acts cell nonautonomously. Close examination of the rescue results and ins-1p::GFP expression in neurons revealed that the defect of the ins-1 mutant was rescued when INS-1 was expressed in at least one of the neurons that appeared to express INS-1 normally (Fig. 2E).

To address the possibility that the lack of rescue by several promoters was caused by either too low or too high expression of the ins-1 cDNA, we constructed ins-1 mutant strains transgenic with different concentrations of ges-1p::ins-1 cDNA or gcy-8p::ins-1 cDNA, and tested those strains for food-associated thermotactic responses. ges-1- or gcy-8-driven INS-1 expression in the ins-1(nr2091) strain did not significantly rescue the defect regardless of the concentration used (Supplementary Fig. 1), suggesting that the inability of some of the promoters to rescue the defect was not caused by insufficient or excessive expression of the ins-1 cDNA.

ins-1 may be regulated transcriptionally, at the hormone processing level, and/or at the level of secretion. We observed whether there was any difference in INS-1 expression between the fed state and the starved state using an ins-1 promoter::GFP fusion gene and a rescuable ins-1::GFP fusion gene. Light microscopic observation failed to find the difference in expression level or localization of the fusion protein (data not shown). These results argue against transcriptional regulation of ins-1 expression. If INS-1 is regulated at the level of secretion in response to starvation, the concentration of extracellular INS-1 may be too low to detect with GFP. Overexpression of INS-1 in a wild-type background induced a partially abnormal thermotaxis phenotype (Fig. 2B), which is consistent with the model that the secretion of INS-1 could modulate thermotactic behavior.

\section{INS-1 antagonizes DAF-2 insulin-like signaling in food-associated thermotactic plasticity}

A previous report suggested that INS-1 antagonizes DAF-2 insulin-like signaling for dauer formation in $C$. elegans (Pierce et al. 2001). We explored whether DAF-2 insulin-like signaling also functions in food-associated thermotactic behavioral plasticity by examining mutants of daf-2, a homolog of the insulin/IGF-1 receptor (Kimura et al. 1997), age-1, a homolog of PI-3-kinase (Morris et al. 1996), and daf-16, a forkhead-type transcriptional factor (Lin et al. 1997). The daf-16(m26) mutant, which is a suppressor of both the daf-2 and the age-1 mutant in dauer formation (Gottlieb and Ruvkun 1994; Larsen et al. 1995), showed a weak Aho phenotype (Fig. 3A). The daf-2(e1368) and age-1(hx546) mutants, however, normally avoided their cultivation temperature after a 3-h starvation (Fig. 3A). If INS-1 antagonizes DAF-2 insulin-like signaling for this integrative behavior, the behavioral responses of daf-2(e1368) and age1(hx546) mutants to starvation might be opposite to the response of ins-1(nr2091) mutants. To test this hypothesis, we conducted a time course assay for starvationinduced temperature avoidance. As the cultivation time under starvation conditions increased, the fraction of wild-type animals that migrated to the cultivation temperature gradually decreased (Fig. 3C). By contrast, the fraction of ins-1(nr2091) mutants that migrated to the cultivation temperature did not decrease much even after a 3-h starvation (Fig. 3C). Consistent with our hypothesis, age-1(hx546) mutants started to avoid the cultivation temperature much earlier than wild-type animals (Fig. 3C). These results indicate that age-1(hx546) could associate cultivation temperature with starvation quicker than wild-type animals. daf-2(e1368) mutants showed a response similar to wild-type animals (Fig. 3C), which might be due to the fact that daf-2(e1368) is one of the weakest alleles in dauer formation /Gems et al. 1998). Because of developmental or behavioral defects, we were unable to examine starvation-induced temperature avoidance of $d a f-2(m g 43)$ and $d a f-2(e 1370)$ mutants, both of which are stronger alleles than daf-2(e1368) in dauer formation (Kimura et al. 1997; Gems et al. 1998; data not shown).

Double mutants were constructed and their food-associated thermotactic responses were tested to clarify the genetic interaction between the insulin-like signaling genes. With a 3 -h starvation, both daf-2 and age-1 mutations partially suppressed the defective starvation-induced temperature avoidance of ins-1 mutants, although daf-2 did not suppress daf-16 (Fig. 3A). These results are consistent with a model that INS-1 acts antagonistically 


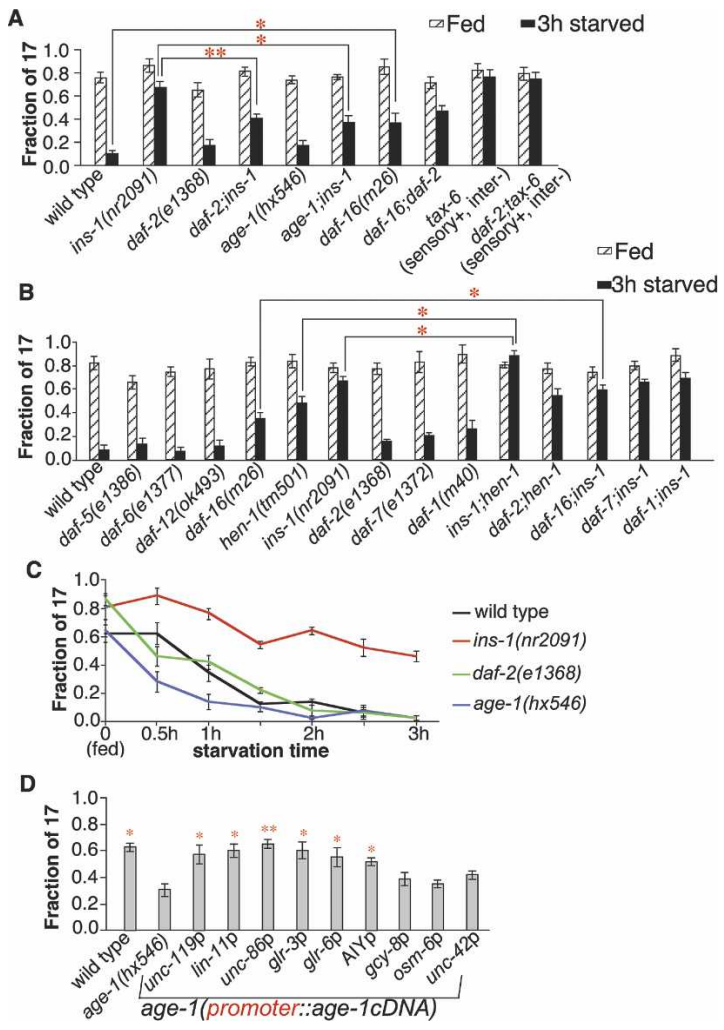

Figure 3. Insulin-like signaling, acting in cooperation with HEN-1, functions in thermotaxis interneurons. $(A, B)$ Results of the thermotaxis assay for well-fed and starved wild-type, single-mutant, and double-mutant animals cultivated at $17^{\circ} \mathrm{C}$. Single and double asterisks indicate $p<0.05$ and $p<0.002$, respectively. Fed animals, $n \geq 40$; starved animals, $n \geq 80$. Error bars indicate SEM. $(C)$ Results of the thermotaxis assay from 0 (fed) to 3-h-starved animals cultivated at $17^{\circ} \mathrm{C}$. Error bars indicate SEM $(n=50$ animals for each time point). ( $D$ ) Rescue experiments of the quicker avoidance defect of the age-1(hx546) mutant. Animals were cultivated under starvation conditions for $30 \mathrm{~min}$ at $17^{\circ} \mathrm{C}$ prior to the thermotaxis assay. Single and double asterisks indicate $p<0.05$ and $p<0.01$, respectively, for a comparison with starved age-1(hx546) mutants ( $n \geq 40$ animals). Error bars indicate SEM.

on DAF-2 insulin-like signaling for food-associated thermotaxis behavior. daf-16; ins-1 double mutants showed a stronger defect than daf-16 single mutants (Fig. 3B), suggesting that ins-1 is genetically downstream from daf-16, which is consistent with a feedback loop from daf-16 to ins-1.

\section{INS-1 and HEN-1 act coordinately in food-associated thermotactic plasticity}

Insulin-like signaling, in cooperation with the TGF- $\beta$ and cyclic GMP pathways, is one of the important pathways for dauer formation. We tested whether dauer formation pathways other than the insulin-like signaling pathway are also involved in integrative behavior for temperature and feeding state. Dauer-defective (Daf-d) mutants for daf-5, daf-6, and daf-12, which encodes the TGF- $\beta$ pathway member Sno/Ski (da Graca et al. 2004), a Patched-related protein that functions upstream of both the TGF- $\beta$ pathway and the cyclic GMP pathway (Schackwitz et al. 1996; Perens and Shaham 2005), and a nuclear receptor (Antebi et al. 2000), respectively, did not show the defects (Fig. 3B). Recently, Murakami et al. (2005) showed that the secreted protein TGF- $\beta / D A F-7$ is involved in memory acquisition of the cultivation temperature. However, daf-7(e1372) and daf-1(m40) mutants that had a deficit in one subunit of the TGF- $\beta$ receptor did not show the defects, and neither the daf-1 nor daf-7 mutant suppressed the defect of ins-1 mutants (Fig. 3B).

HEN-1, a secretory protein with an LDL receptor motif, is reported to be involved in food-associated thermotactic plasticity (Ishihara et al. 2002). To test for a genetic interaction between insulin-like signaling and HEN-1, we constructed double mutants. daf-2 mutation did not suppress the defect of hen-1 mutants. The ins-1; hen-1 double mutant, however, showed a stronger mutant phenotype than ins-1 or hen-1 single mutants (Fig. $3 \mathrm{~B})$. These results suggest that INS-1 and HEN-1 act in parallel and that insulin-like signaling and HEN-1 signaling are major components in the regulation of foodassociated thermotactic plasticity.

\section{Insulin-like signaling functions in thermotaxis interneurons}

Where is the target cell that receives and processes INS-1 to antagonize the DAF-2 insulin-like signaling pathway for temperature-starvation integrative behavior? To address this question, we conducted a cell-specific rescue experiment on age-1(hx546) mutants with a 30-min starvation. Expressing age-1 cDNA in all neurons using the unc-119 promoter rescued the quicker starvation-induced temperature avoidance defect of age-1(hx546) mutants (Fig. 3D). Expressing age-1 cDNA in several neurons, including the AIZ, AIY, or RIA interneurons, all of which are essential interneurons for thermotaxis (Mori and Ohshima 1995), almost fully rescued the quicker avoidance defect of age-1(hx546) mutants (cf. Figs. 3D and 2E; Brockie et al. 2001). By contrast, expressing age-1 cDNA in AFD thermosensory neurons by the gcy-8 promoter, in sensory neurons by the osm-6 promoter or in many neurons by the unc-42 promoter did not rescue the defect (cf. Figs. 3D and 2E). These results suggest that AGE-1 (and probably the insulin-like signaling pathway) functions in thermotaxis interneurons for food-associated neural plasticity.

\section{Calcium imaging of the thermotaxis interneurons}

To analyze a physiological aspect of the insulin-like signaling in integrative behavior for food and temperature, we observed the changes in neuronal activity of the AIZ thermotaxis interneuron of live animals by measuring stimulus-evoked $\mathrm{Ca}^{2+}$ concentration changes using cameleon, a genetically encodable calcium indicator (Miyawaki et al. 1997; Kimura et al. 2004). The activity of the AIZ interneuron in wild-type animals cultivated at $17^{\circ} \mathrm{C}$ with food increased with warming and decreased with cooling, whereas the activity of the AIZ interneuron in the starved wild-type animals was much less responsive to temperature changes (Fig. 4A; Kuhara and Mori 2006). The AIZ interneuron of starved ins-1(nr2091) animals was as active as that of fed ins-1(nr2091) animals (Fig. 4B). These results suggest that INS-1 is required for the starvation-induced negative regulation of AIZ neuron activity. Calcium imaging on AFD thermosensory neurons revealed that feeding state did not influence the activity of AFD (Fig. 4C). 
A

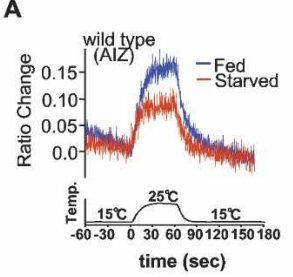

B

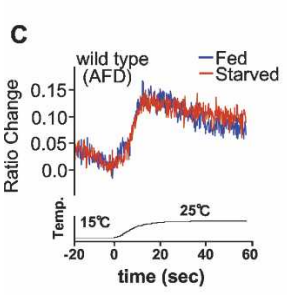

D
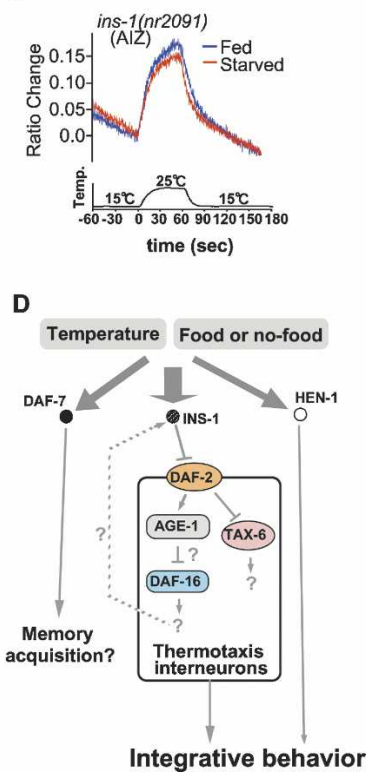

Figure 4. In vivo calcium imaging and model for food-associated thermotactic behavioral plasticity. $(A, B)$ Calcium imaging of the AIZ interneuron in animals cultivated at $17^{\circ} \mathrm{C}$ under fed or starved conditions expressing the cameleon protein. $(A)$ Wild-type animals. (B) ins-1(nr2091) mutants. $n=10-13$. Relative increases or decreases in the intracellular calcium concentration were measured as increases or decreases, respectively, in the YFP/CFP fluorescence ratio of the cameleon protein (Ratio Change). Temperature (Temp.) is shown as a black line at the bottom. (C) Calcium imaging of the AFD thermosensory neuron in wild-type animals grown at $20^{\circ} \mathrm{C}$ expressing the cameleon protein cultivated under fed or starved conditions $(n=18-20)$. Temperature (Temp.) is shown as a black line at the bottom. (D) Model of the suggestive genetic pathway for modulation of integrative behavior between cultivation temperature and feeding state by insulin-like signaling based on the results presented in this study. DAF-7 is thought to be involved in memory acquisition of cultivation temperature (Murakami et al. 2005).

We recently found that the interneuron-deficient calcineurin mutant tax-6(sensory+, inter-) also showed a defect in association between temperature and feeding state at $17^{\circ} \mathrm{C}$ (Fig. $3 \mathrm{~A}$; Kuhara and Mori 2006). TAX-6 calcineurin is required for temperature-starvation integrative behavior in both of two directly connected interneurons, AIZ and RIA, and like INS-1, TAX-6 is required for starvation-induced regulation of AIZ activity (Kuhara and Mori 2006). To investigate the genetic interaction between calcineurin-mediated signaling and insulin-like signaling in thermotaxis interneurons, we constructed daf-2; tax-6(sensory+, inter-) mutants. The food-associated thermotactic behavior defect of the tax-6/sensory+, inter-) mutation was not suppressed by the daf-2 mutation (Fig. 3A), which is consistent with the possibility that TAX-6 calcineurin acts downstream from DAF-2 in thermotaxis interneurons. We also investigated whether DAF-16 is involved in transcription of tax-6 by comparing the expression of a tax- $6:$ GFP translational fusion gene in a wild-type background with expression of the fusion gene in a daf-16(mu86) deletion mutant background. We could not find any differences in expression level (data not shown). These results are consistent with the possibility that daf-16 is not required for the transcription of tax-6.

A neuroendocrine system modulates the neural circuit important for integrative behavior

Our results propose a model for food-associated thermotactic plasticity (Fig. 4D). During association between cultivation temperature and starvation, INS-1 is secreted from several neurons and antagonizes insulin-like signaling by inhibiting the activity of DAF-2 receptor. DAF16 may be activated, probably through AGE-1, and a feedback loop from DAF-16 to INS-1 might exist. HEN-1 might also be secreted from AIY or ASE neurons (Ishihara et al. 2002). We thus suggest that a neuroendocrine system is important for modulating the neural circuit that underlies the integrative behavior.

Murakami et al. (2005) argued that AGE-1 acts in AIY neurons to enhance isothermal tracking, which is one aspect of thermotaxis. Insulin-like signaling is required for salt chemotaxis learning, where animals pre-exposed to the chemoattractant $\mathrm{NaCl}$ under starvation condition exhibit reduced chemotactic response to $\mathrm{NaCl}$ (Tomioka et al. 2006). These reports also implicate the importance of insulin-like signaling in behavioral plasticity.

What are the targets of DAF-16 in integrative behavior for temperature and food? Insulin-like signaling is a part of the dauer formation pathway, which has many feedback loops (Schackwitz et al. 1996). The results of the present study are consistent with a feedback loop in insulin-like signaling for this integrative behavior. Assuming the existence of a feedback loop, one clue for the targets of DAF-16 in the thermotactic plasticity might be found in a report by Murphy et al. (2003), which suggested that the insulin homologs INS-2, INS-7, INS-18, and INS-21 are likely to be direct or indirect targets of DAF-16. Likewise, it is plausible that any of these insulin-like molecules act agonistically on DAF-2 to activate the insulin-like pathway in food-associated thermotactic plasticity. We believe that these issues are critical for further study.

\section{Materials and methods}

Strains and genetics

The standard techniques were used for culturing and handling C. elegans. For details and strains, see Supplemental Material.

\section{Behavioral assays}

A radial temperature-gradient assay was performed as described previously (Mori and Ohshima 1995; Mohri et al. 2005). The locomotory rate assay was performed according to a previous report (Sawin et al. 2000). The interaction assay was also performed as previously described (Ishihara et al. 2002). For details, see Supplemental Material.

Molecular biology and germline transformation

Standard methods for molecular biology and germline transformation were used. For details and vectors, see Supplemental Material.

In vivo calcium imaging and data analysis

In vivo calcium imaging was performed essentially according to Kimura et al. (2004) and Kuhara and Mori (2006). For details, see Supplemental Material.

\section{Acknowledgments}

We thank G. Ruvkun, C. Wolkow, P. Hu, and J. Xu for kindly providing ins-1(nr2091), daf-2(mg43), and age-1 DNAs and age-1(mg305) mutants; T. Ishihara for hen-1(tm501) mutants; A. Fire for pPD plasmids; O. Hobert and Y. Okochi for the AIY and lin-11 promoters; B. Wedel, D. Garbers, and H. Inada for the gcy-8 promoter; Y. Tanizawa for the glr-3 
short promoter; P. Swoboda and H. Sasakura for the osm-6 promoter; J. Sze for the tph-1 promoter; J. McGhee for the ges-1 promoter; H. Kagoshima for the ceh-14 promoter; all the members of Mori Laboratory for technical advice and stimulating discussions; A. Coulson and R. Shownkeen for cosmids; and the C. elegans Sequence Consortium for updating the C. elegans genome information. The Caenorhabditis Genetic Center provided some of the strains used in this study. This work was supported by Grant-in-Aid for Scientific Research on Priority Areas-Molecular Brain Science from the MEXT (00210010) and by HFSPO (to I.M.). I.M. is a Scholar of the Institute for Advanced Research of Nagoya University.

\section{References}

Abbott, M.A., Wells, D.G., and Fallon, J.R. 1999. The insulin receptor tyrosine kinase substrate $\mathrm{p} 58 / 53$ and the insulin receptor are components of CNS synapses. J. Neurosci. 19: 7300-7308.

Antebi, A., Yeh, W., Tait, D., Hedgecock, E.M., and Riddle, D.L. 2000 daf-12 encodes a nuclear receptor that regulates the dauer diapause and developmental age in C. elegans. Genes \& Dev. 14: 1512-1527.

Brockie, P.J., Madsen, D.M., Zheng, Y., Mellem, J., and Maricq, A.V 2001. Differential expression of glutamate receptor subunits in the nervous sytem of Caenorhabditis elegans and their regulation by the homeodomain protein UNC-42. J. Neurosci. 21: 1510-1522.

da Graca, L.S., Zimmerman, K.K., Mitchell, M.C., Kozhan-Gorodetska M., Sekiewicz, K., Morales, Y., and Patterson, G.I. 2004. DAF-5 is a Ski oncoprotein homolog that functions in a neuronal TGF $\beta$ pathway to regulate C. elegans dauer development. Development 131: 435446.

Gems, D., Sutton, A.J., Sundermeyer, M.L., Albert, P.S., King, K.V., Edgley, M.L., Larsen, P.L., and Riddle, D.L. 1998. Two pleiotropic classes of daf-2 mutation affect larval arrest, adult behavior, reproduction and longevity in Caenorhabditis elegans. Genetics 150: 129-155.

Gottlieb, S. and Ruvkun, G. 1994. daf-2, daf-16 and daf-23: Genetically interacting genes controlling dauer formation Caenorhabditis elegans. Genetics 137: 107-120.

Havrankova, J., Roth, J., and Brownstein, M. 1978a. Insulin receptors are widely distributed in the central nervous system of the rat. Nature 272: 827-829.

Havrankova, J., Schmechel, D., Roth, J., and Brownstein, M. 1978b. Identification of insulin in rat brain. Proc. Natl. Acad. Sci. 75: 5737-5741.

Hedgecock, E.M. and Russell, R.L. 1975. Normal and mutant thermotaxis in the nematode Caenorhabditis elegans. Proc. Natl. Acad. Sci. 72: 4061-4065.

Inada, H., Ito, H., Satterlee, J., Sengupta, P., Matsumoto, K., and Mori, I. 2006. Identification of guanylyl cyclases that function in thermosensory neurons of Caenorhabditis elegans. Genetics 172: 2239-2252.

Ishihara, T., Iino, Y., Mohri, A., Mori, I., Gengyo-Ando, K., Mitani, S., and Katsura, I. 2002. HEN-1, a secretory protein with an LDL receptor motif, regulates sensory integration and learning in Caenorhabditis elegans. Cell 109: 639-649.

Kelly, A. and Lynch, M.A. 2000. Long-term potentiation in dentate gyrus of the rat is inhibited by the phosphoinositide 3-kinase inhibitor, wortmannin. Neuropharmacology 39: 643-651.

Kimura, K.D., Tissenbaum, H.A., Liu, Y., and Ruvkun, G. 1997. daf-2, an insulin receptor-like gene that regulates longevity and diapause in Caenorhabditis elegans. Science 277: 942-946.

Kimura, K.D., Miyawaki, A., Matsumoto, K., and Mori, I. 2004. The $C$. elegans thermosensory neuron AFD responds to warming. Curr. Biol. 14: 1291-1295.

Komatsu, H., Mori, I., Rhee, J.S., Akaike, N., and Ohshima, Y. 1996. Mutations in a cyclic nucleotide-gated channel lead to abnormal thermosensation and chemosensation in C. elegans. Neuron 17: 707718.

Kuhara, A. and Mori, I. 2006. Molecular physiology of the neural circuit for calcineurin-dependent associative learning in Caenorhabditis elegans. J. Neurosci. 26: 9355-9364.

Kuhara, A., Inada, H., Katsura, I., and Mori, I. 2002. Negative regulation and gain control of sensory neurons by the $C$. elegans calcineurin TAX-6. Neuron 33: 751-763.

Larsen, P.L., Albert, P.S., and Riddle, D.L. 1995. Genes that regulate both development and longevity in Caenorhabditis elegans. Genetics 139: $1567-1583$.

Li, W., Kennedy, S.G., and Ruvkun, G. 2003. daf-28 encodes a C. elegans insulin superfamily member that is regulated by environmental cues and acts in the DAF-2 signaling pathway. Genes \& Dev. 17: 844-858.

Lin, K., Dorman, J.B., Rodan, A., and Kenyon, C. 1997. daf-16: An HNF3/forkhead family member that can function to double the life-span of Caenorhabditis elegans. Science 278: 1319-1322.

Man, H.Y., Lin, J.W., Ju, W.H., Ahmadian, G., Liu, L., Becker, L.E., Sheng, M., and Wang, Y.T. 2000. Regulation of AMPA receptor-mediated synaptic transmission by clathrin-dependent receptor internalization. Neuron 25: 649-662.

Miyawaki, A., Llopis, J., Heim, R., McCaffery, J.M., Adams, J.A., Ikura, M., and Tsien, R.Y. 1997. Fluorescent indicators for $\mathrm{Ca}^{2+}$ based on green fluorescent proteins and calmodulin. Nature 388: 882-887.

Mohri, A., Kodama, E., Kimura, K.D., Koike, M., Mizuno, T., and Mori, I. 2005. Genetic control of temperature preference in the nematode Caenorhabditis elegans. Genetics 169: 1437-1450.

Mori, I. and Ohshima, Y. 1995. Neural regulation of thermotaxis in Caenorhabditis elegans. Nature 376: 344-348.

Morris, J.Z., Tissenbaum, H.A., and Ruvkun, G. 1996. A phosphatidylinositol-3-OH kinase family member regulating longevity and diapause in Caenorhabditis elegans. Nature 382: 536-539.

Murakami, H., Bessinger, K., Hellmann, J., and Murakami, S. 2005. Aging-dependent and -independent modulation of associative learning behavior by insulin/insulin-like growth factor-1 signal in Caenorhabditis elegans. J. Neurosci. 25: 10894-10904.

Murphy, C.T., McCarroll, S.A., Bargmann, C.I., Fraser, A.F., Kamath, R.S., Ahringer, J., Li, H., and Kenyon, C. 2003. Genes that act downstream of DAF-16 to influence the lifespan of Caenorhabditis e1 egans. Nature 424: 277-284.

Okochi, Y., Kimura, K.D., Ohta, A., and Mori, I. 2005. Diverse regulation of sensory signaling by C. elegans nPKC-E/eta TTX-4. EMBO J. 24: 2127-2137.

Perens, E.A. and Shaham, S. 2005. C. elegans daf-6 encodes a patchedrelated protein required for lumen formation. Dev. Cell 8: 893-906.

Pierce, S.B., Costa, M., Wisotzkey, R., Devadhar, S., Homburger, S.A., Buchman, A.R., Ferguson, K.C., Heller, J., Platt, D.M., Pasquinelli, A.A., et al. 2001. Regulation of DAF-2 receptor signaling by human insulin and ins-1, a member of the unusually large and diverse $C$. elegans insulin gene family. Genes \& Dev. 15: 672-686.

Rankin, C.H. 2005. Nematode memory: Now, where was I? Curr. Biol. 15: R374-R375.

Sawin, E.R., Ranganathan, R., and Horvitz, H.R. 2000. C. elegans locomotory rate is modulated by the environment through a dopaminergic pathway and by experience through a serotonergic pathway. Neuron 26: 619-631.

Schackwitz, W.S., Inoue, T., and Thomas, J.H. 1996. Chemosensory neurons function in parallel to mediate a pheromone response in C. elegans. Neuron 17: 719-728.

Tomioka, M., Adachi, T., Suzuki, H., Kunitomo, H., Schafer, W.R., and Iino, Y. 2006. The insulin/PI 3-kinase pathway regulates salt chemotaxis learning in Caenorhabditis elegans. Neuron 51: 613-625.

White, J.G., Southgate, E., Thomson, J.N., and Brenner, S. 1986. The structure of the nervous system of the nematode Caenorhabditis elegans. Philos. Trans. R. Soc. Lond. B Biol. Sci. 314: 1-340. 


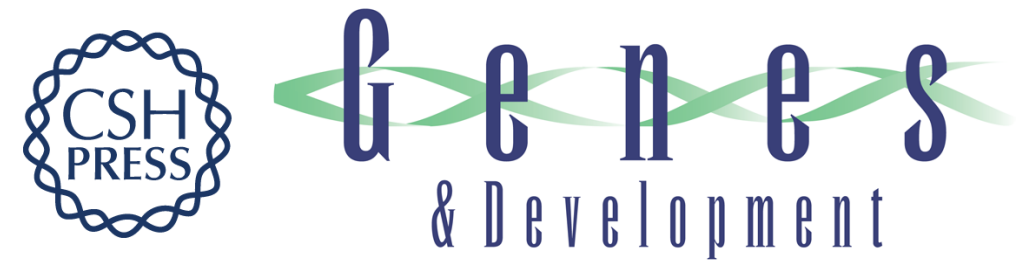

\section{Insulin-like signaling and the neural circuit for integrative behavior in C. elegans}

Eiji Kodama, Atsushi Kuhara, Akiko Mohri-Shiomi, et al.

Genes Dev. 2006, 20:

Access the most recent version at doi:10.1101/gad.1479906

\section{Supplemental http://genesdev.cshlp.org/content/suppl/2006/10/20/20.21.2955.DC1 Material}

References This article cites 35 articles, 17 of which can be accessed free at: http://genesdev.cshlp.org/content/20/21/2955.full.html\#ref-list-1

\section{License}

Email Alerting

Receive free email alerts when new articles cite this article - sign up in the box at the top Service 